\title{
Zuviel Angst vor heterodoxen Schulen kann medizinische Innovation verhindern
}

\author{
M. Hänggi *
}

\footnotetext{
* Marcel Hänggi, Wissenschaftsredaktor der «WOZ Die Wochenzeitung», hat über die Einführung der Anästhesie eine Lizentiatsarbeit geschrieben, die in der Bibliothek des medizinhistorischen Instituts der Universität Zürich eingesehen werden kann [1].
}

a Die meines Erachtens beste Geschichte des Schmerzes stammt von Roselyne Rey [4].

1 Hänggi M. Die Anfänge der Anästhesie in der Schweiz (1847). Zürich 2002, unveröffentlicht.

2 Geiser M. Das Ansehen nicht nur der FMH steht auf dem Spiel. Schweiz Ärztezeitung 2005; 86(8):470-2

3 Davy H. Researches, Chemical and Philosophical, Chiefly Concerning Nitrous Oxide or Dephlogisticated Nitrous Air and its Respiration. London: J. Johnson; 1800.

4 Rey R. Histoire de la douleur: Paris: Editions la Découverte; 1993.

5 NZZ vom 20. Januar 1847.

Korrespondenz:

Marcel Hänggi, lic. phil. / Journalist BR Hofstrasse 16

CH-8032 Zürich

Tel. 0442523923

E-Mail: marcel.haenggi@schweiz.ch
In der Schweizerischen Ärztezeitung Nr. 8/2005 hofft der Autor M. Geiser, die Öffentlichkeit möge «die jämmerliche Wirkungslosigkeit der Heilkunde bis zu Beginn des 19. Jahrhunderts und die Tatsache zur Kenntnis [nehmen], dass die Revolutionierung der Medizin zur effektvollen biomedizinischen Wissenschaft durch die Beseitigung von Dogmata vollzogen wurde.» Geisers Botschaft lautet: Hände weg von allem Unwissenschaftlichen, sprich: von der Komplementärmedizin [2].

Ganz so eindeutig ist die Lektion der Geschichte freilich nicht. Eine der - aus heutiger Sicht - segensreichsten Neuerungen der Medizin im 19. Jahrhundert, die Anästhesie, hätte viel früher schon «entdeckt» werden können, hätten die damals tonangebenden Mediziner etwas weniger Furcht vor heterodoxen Methoden gehabt.

Im allgemeinen gilt der 13. Oktober 1846 (der «Ether-Day») als Geburtstag der Anästhesie. An dem Tag exstirpierte der Zahnarzt und Chirurg Thomas G. Morton vor medizinischem Fachpublikum am Bostoner Universitätsspital den Halstumor eines Patienten, den er zuvor mittels Inhalation von Schwefeläther narkotisiert hatte. Allerdings: Bereits 1800 hatte der junge britische Arzt Humphrey Davy vorgeschlagen, ein Gas - Lachgas - zur Schmerzbekämpfung bei chirurgischen Operationen anzuwenden [3].

\section{«Unseriöse» Herkunft}

Wissenschaftsgeschichte lässt sich nicht einfach als siegreicher Kampf der Erkenntnis gegen die Unwissenheit verstehen. Ob sich eine Erkenntnis durchsetzt, hängt immer auch von sozialen und anderen wissenschaftsexternen Faktoren ab. Als Davy seine bewusstseinserweiternden «pneumatischen» Experimente machte, lebte er ein Dandyleben; an seinen Versuchen beteiligten sich Schriftsteller wie Samuel T. Coleridge oder William Wordsworth. Das waren nicht unbedingt die Voraussetzungen, um sich in der medi- zinischen Wissenschaft durchzusetzen, die sich um 1800 zunehmend akademisch zu organisieren und gegen alles «Unseriöse» abzugrenzen begann. Lachgas machte seine Karriere nicht in Operationssälen, sondern an Partys. Später arrivierte Davy zum Vorsitzenden der britischen Royal Society. Und unter seiner Führung lehnt es diese 1823 ab, Versuche, mittels Gasinhalation schmerzfrei zu operieren, auch nur zu prüfen. Angelangt im Zentrum des akademischen Establishments, konnte Davy es sich nicht mehr leisten, sich mit Experimenten abzugeben, die er als junger Mann selber angestellt hatte. Auch die französische Académie Royale de Médecine wollte nichts von Anästhesieversuchen wissen.

Das lange Zögern hat mannigfaltige Gründe und hat unter anderem mit dem Verständnis von Schmerz zu tun. ${ }^{a}$ Hinweise auf einen weiteren möglichen Grund für die offensichtliche Scheu der akademischen Medizin vor der Anästhesie geben die ersten Reaktionen auf den «EtherDay». «Gentlemen, dies ist kein Humbug!» soll John C. Warren, der Morton zu seiner Demonstration eingeladen hatte, nach erfolgreicher Operation gesagt haben. «Diese Glanzidee der Yankees, meine Herren, ist der Hypnose haushoch überlegen. Welch ein Glück!», rief der Londoner Chirurg Joseph Liston aus, der die neue Methode als erster in Europa anwandte. Die NZZ betonte, es handle sich «nicht etwa um magnetische Einschläferung des Patienten» [5]. «Humbug»: Das war es, was Vertreter der orthodoxen Medizin bis 1846 mit der Idee der Schmerzausschaltung assoziierten; «Hypnose» und «magnetische Einschläferung» waren praktisch Synonyme dafür.

\section{«Animalischer Magnetismus»}

Was hiess «magnetische Einschläferung»? Im 18. Jahrhundert postulierte Franz Anton Mesmer, Tiere und Menschen wiesen ähnlich wie Eisengegenstände einen Magnetismus auf, der sich durch Überstreichungen ausrichten liesse. 
Mesmer behandelte verschiedenste Krankheiten nach seiner Methode; zu seinen Anhängern gehörten einflussreiche Personen bis hin zu Königin Marie-Antoinette.

Der Mesmerismus erfreute sich nach Mesmers Tod in der ersten Hälfte des 19. Jahrhunderts in London grosser Beliebtheit, wie Alison Winter in einem hervorragenden Buch darstellt [6]. Während in der Schulmedizin die Definitionsmacht über Krankheit immer stärker vom Patienten auf den Arzt überging, bo überliess der Magnetiseur die Diagnose und teilweise sogar die Festsetzung einer Therapie seinem Patienten im Trancezustand.c Trotz dieses Anachronismus stand die einflussreiche Fachzeitschrift «The Lancet» dem Mesmerismus wohlwollend gegenüber - bis 1838, als bei öffentlichen mesmeristischen Demonstrationen dem behandelnden Arzt die Kontrolle über seine Patientin entglitt (diese beschimpfte die anwesenden Honoratioren obszön). «The Lancet» wurde zum wütenden Kampfblatt wider den Mesmerismus.

Seit den 1820er Jahren verfügten Mesmeristen aber über einen besonderen Trumpf. Sie hatten festgestellt, dass der «Magnetschlaf» bei Patienten die Schmerzempfindung ausschalten konnte (heute erklärt man sich dieses Phänomen in der Regel mit einem Hypnoseeffekt). 1842 amputierte William Topham vor Fachpublikum einen Oberschenkel. Statt zu schreien, murmelte der Patient lediglich und gab nach Erwachen an, keinen Schmerz verspürt zu haben. Die Zuschauer aber glaubten nicht, was sie gesehen hatten. Man traute dem Patienten zu, seine Empfindungslosigkeit simuliert $\mathrm{zu}$ haben! ${ }^{\mathrm{d}}$ Wer etwas auf seinen akademischen Ruf gab, wollte mit der Lehre Mesmers nichts zu tun haben.

Nun mehrten sich aber die Berichte erfolgreicher schmerzfreier Operationen nach «Magnetisierung» des Patienten. Der Mesmerismus zeigte immer deutlicher, dass er der Schulmedizin gefährlich werden konnte. Die Entdeckung der Inhalationsanästhesie gab der Schulmedizin endlich ein Mittel gegen die mesmeristische Konkurrenz in die Hand. So erklärt sich, dass Liston nach seiner ersten Operation unter Äthernarkose mehr den Sieg über die «Hypnose» feierte als den Sieg über den Schmerz. Für diesen Sieg hatte es aber zuerst eines Zahnarztes in den USA - also eines Handwerkers in einem Land, wo die medizinische Zunft viel weniger straff organisiert war als in Europa - bedurft, der keinen akademischen Ruf zu verlieren hatte.

\section{Eine Lanze gegen die Mesmeristen}

Dass die Anästhesie in der europäischen Schulmedizin praktisch sofort etabliert war, war nicht zuletzt ein Verdienst des «Lancet», das im ersten Halbjahr 1847 über hundert Beiträge zum Thema veröffentlichte. Der Mesmerismus spielte mithin eine paradoxe Rolle in der Geschichte der Anästhesie: Das Bestreben, nichts mit der «Irrlehre» zu tun haben zu wollen, verzögerte den Einzug der Anästhesie in die Schulmedizin bis 1846; die Tatsache, dass man dem Mesmerismus mit der Inhalationsanästhesie den Boden unter den Füssen wegziehen konnte, beschleunigte deren Verbreitung ab 1847. Mit anderen Worten: Die orthodoxe Medizin akzeptierte die Anästhesie zu dem Zeitpunkt, wo sie sich nicht länger dagegen wehren konnte, ohne allzuviel Terrain an ihren Konkurrenten, den Mesmerismus, zu verlieren. Dann aber eignete sie sich die Innovation - die man wissenschaftlich überhaupt nicht verstand - sofort an und verwendete sie gegen ebendiesen Konkurrenten. Die Rechnung ging auf: Wenige Jahre später war diese Lehre praktisch vergessen.

Ein interessanter Nebenaspekt der Geschichte ist der kommerzielle: Morton versuchte, die Zusammensetzung seines Gases geheimzuhalten, um es patentieren zu lassen, doch der Geruch verriet ihn. Ein heftiger Prioritätenstreit entbrannte zwischen ihm und seinem Widersacher Horace Wells. Die wirtschaftliche Bedeutung war um so grösser, als wohl zum ersten Mal in der Medizingeschichte die Publikumszeitungen eine wichtige Rolle bei der Verbreitung einer Neuerung spielten. Johann Jakob Jenny, Pionier der Anästhesie in der Schweiz aus Ennenda, bastelte seinen ersten Inhalationsapparat aus einer Schweinsblase aufgrund von Zeitungsberichten, und in der NZZ tauchten wenige Monate nach Bekanntwerden der Anästhesie schon Annoncen für Inhalationsapparate auf.

Auch die Zürcher Ärzte erkannten die wirtschaftliche Bedeutung der Errungenschaft bald: Sie kümmerten sich als erstes darum, sich das Monopol auf die neue Methode zu sichern. In einem Erlass der kantonalen Sanitätskommission, die Conrad Meyer-Hoffmeister, Sekundärarzt am Universitätsspital, der Kommission wörtlich diktiert hatte, wurde allem nichtärztlichen Medizinpersonal die Anwendung der Anästhesie verboten. Die Ärzteschaft nutzte die neue Errungenschaft - keinen Monat nach der ersten Operation unter Äthernarkose im Kanton -, um ihre Konkurrenz zurückzubinden. 